\title{
Transfer Learning for QoS Aware Topology Management in Energy Efficient 5G Cognitive Radio Networks
}

\author{
Qiyang Zhao and David Grace \\ Department of Electronics, University of York, York, United Kingdom \\ \{qiyang.zhao,david.grace\}@york.ac.uk
}

\begin{abstract}
In this paper, we investigate the use of a transfer learning approach applied to a topology management framework in a 5G heterogeneous aerial-terrestrial broadband access network, to reduce energy consumption and deployment cost, and improve system capacity and QoS. We implement a cognitive engine at the base station (BS), with reinforcement learning algorithms applied at the link level for spectrum assignment, and at the network level for user association. A novel transfer learning algorithm is developed to transfer the expertise knowledge learnt from spectrum assignment to formulate a knowledgebase for user association. Furthermore, a QoS aware base station switching operation algorithm is proposed at a network controller, to dynamically switch BSs between sleep and active modes based on system QoS requirements. System simulations under practical configurations show that the transfer learning based user association algorithm achieves significant energy saving and QoS improvement with optimized load management in a spectrum sharing scenario. The BS switching operation algorithm effectively controls the delay and retransmissions when saving energy from sleep mode.
\end{abstract}

Keywords - Transfer Learning, Energy Efficient 5G, User Association, Sleep Mode

\section{INTRODUCTION}

The future "fifth generation (5G)" wireless communication system has been proposed to provide a high capacity density broadband access service to mobile users. To achieve this, a dense deployment of light-weight small cell Base Stations (BSs) have been considered as an effective approach, which is able to enhance local capacity and improve Quality of Service (QoS) in the area with high user or traffic density [1]. In this context, small cells are usually deployed in hotspot areas under macrocells which provide coverage. A heterogeneous macrosmall cell architecture can largely improve spectrum efficiency and reduce the cost of network deployment and operation.

Energy consumption in the Radio Access Network (RAN) is one of the key challenges in future $5 \mathrm{G}$ infrastructure, and there is an aim to save up to $90 \%$ of energy used per service provided compared with today [2]. Currently, the electricity consumption from wireless network accounts for around $2 \%$ of the global $\mathrm{CO}_{2}$ emissions, and $75 \%$ of the energy comes from the BS side [3]. Moreover, in many situations two thirds of the energy in a BS is consumed by the power amplifiers and air conditioning to keep the BS working. In this context, the required dense deployment of small cell BSs may cause severe challenges in terms of energy consumption. This is despite the fact that small cells BSs have a reduced transmit power compared with macro cell deployments, and the energy to deliver the required transmit power is only a small proportion of the total energy consumption [4]. A study of power models of LTE eNBs by the FP7 EARTH project shows that in a
Micro, Pico or Femto cell, the base band and RF components dominate the overall power consumption [5].

Traffic aware RAN management has been a hot research topic, which aims at reducing energy consumption from optimized network planning and deployment, or dynamic BS sleep mode operation. [6] investigates the impact of BS deployment on system throughput and power consumption. This is further improved in [7] which optimizes the location of BS considering spatial user traffic distribution. Sleep modes have been introduced in $[3,8]$ which save energy from traffic variation in the time domain, and a load aware BS switching operation algorithm has been proposed in [9] based on this paradigm. On the other hand, the effect of user association and load balancing on energy and delay has been studied in [10], and QoS aware BS switching operation and user association algorithms have been proposed in [11]. Most of these approaches are based on instant or short term monitoring of system parameters, i.e. traffic load, delay, and blocking probability. However, in practice the user behaviour and radio environment is highly dynamic, which causes excessive variations on these parameters. Under this effect, the system cannot evaluate the stability of the environment and make effective decisions on switching operation or user association, which in turn reduce QoS and energy efficiency.

In this paper, we formulate BS switching operation and user association collectively as topology management, and introduce the cognitive radio technology to deal with this problem in a dynamic radio spectrum and user traffic environment. Future $5 \mathrm{G}$ networks are likely to introduce dense small cells and dynamic spectrum access (DSA) to enhance system capacity density. In this context, a user is likely to have multiple BSs to connect to, and these BSs are allowed to share a common spectrum band. Thus in the 5G network, user association and spectrum assignment will need to be highly flexible, and will also be subject to severe challenges, such as interference and traffic congestion. Conventional centralized or decentralized algorithms become complex and ineffective, and cannot satisfy the self-organization requirement in $5 \mathrm{G}$ architecture design. In order to make user association and spectrum assignment distributed and intelligent, we apply cognitive radio engines to these two domains. It allows the BSs to learn the radio environment and user behaviour, and make effective decisions.

The target of using cognitive radio on user association and spectrum assignment is to achieve effective QoS and energy saving. From the radio resource management perspective, these two domains are affected by similar factors, such as interference and spectrum availability. In this context, the knowledge learnt from one domain can potentially be used on the other. Therefore, we can deal with this issue by employing 
the conceptual idea of transfer learning (TL). Transfer learning was originally proposed by the computer science community [12] as a method of improving learning in the new target task by transferring knowledge from the related source tasks. It has been investigated in [13] for BS switching operation. However, a major issue of applying learning to switching operation is that the BSs can be switched on and off frequently at the initial stage of learning in order to train the knowledgebase using trial-and-error operation. This is inefficient in practice where the time required for a BS to switch on/off is significantly longer than a user arrival/departure. On the other hand, the amount of energy saving from switching operation is ultimately determined by system QoS and capacity, where user association and spectrum assignment have major impact. As a result, we apply transfer learning to these two highly related domains. More importantly, they are carried out for every data file transmission in any cellular system, meaning that the learning algorithm can obtain sufficiently large amount of trails without disrupting the radio system.

The remainder of this paper is organized as follows. Section II describes the aerial-terrestrial broadband access network architecture. In section III, the QoS aware topology management approaches are investigated, followed by a transfer learning algorithm designed for spectrum assignment and user association. Section IV presents the system level simulation and discusses the results. We finally conclude this paper in Section V.

\section{SYSTEM MODEL AND ARCHITECTURE}

In this paper, we investigate a heterogeneous aerialterrestrial broadband access network proposed by the FP7 ABSOLUTE project [14]. It is constructed from a new design of two types LTE eNBs: an Aerial eNB (AeNB) on a Low Altitude Platform (LAP) which provides coverage over a large area, and a portable Terrestrial eNB (TeNB) which enhances network capacity in hotspot areas. The system is designed to provide reliable LTE services in various scenarios with significantly different user requirements, such as disaster relief and temporary events. The AeNBs can provide high data rate links over wide rural and remote areas. A dense deployment of TeNBs can provide a high capacity density network in urban areas. The full system architecture including both access and satellite backhaul links is demonstrated in Fig. 1.

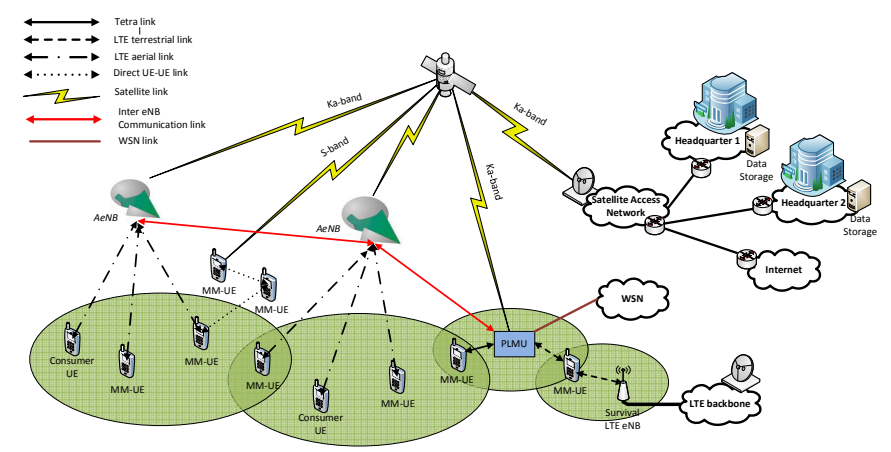

Fig. 1. Aerial-Terrestrial Broadband Network Architecture

In order to investigate and develop energy saving solutions that are generally applicable to a heterogeneous macro-small cell scenario, we hereby consider the LTE access network in this architecture. In practical networks eNBs will be interspaced at intervals in suitable locations. Here, an example system model for the access network is demonstrated in Fig. 2, which simulates a $2.4 \mathrm{~km}^{2}$ rectangular urban area.

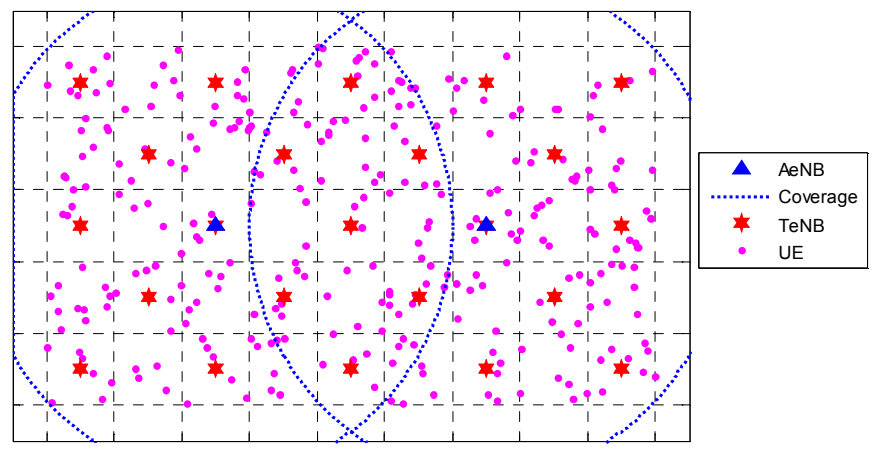

Fig. 2. Example Aerial-Terrestrial Broadband Access Network Model

A total number of 2 AeNBs and 23 TeNBs are considered and modelled on a grid for convenience and ease of interpretation of results. There are 300 UEs randomly placed in the area which have access to both AeNBs and TeNBs. The entire system is allocated with $20 \mathrm{MHz}$ spectrum, which is equally divided into 2 bands for AeNBs and TeNBs. In each band the eNBs share the entire $10 \mathrm{MHz}$ spectrum.

The AeNBs and TeNBs are light-weight equipment and each of which has $5 \mathrm{~W}$ transmit power. The microcell energy model from the FP7 EARTH project [5] is used to evaluate their power consumption. The total power consumed by all eNBs in the network is

$$
P=\frac{\sum_{i=1}^{n_{\text {oNBS }}} N_{T R X}\left(\Delta_{P} P_{\text {outi, }}\left(t_{T x, i}+t_{R x, i}\right)+P_{0, i}\left(t_{T x, i}+t_{R x, i}+t_{0, i}\right)+P_{s} t_{s, i}\right)}{t_{T x}+t_{R x}+t_{0}+t_{s}}
$$

where $P_{\text {out }}$ denotes the RF output power at maximum load, $P_{0}$ is the linear model parameter to represent power consumption at zero RF output power and $\Delta_{P}$ is the slope of the load dependent power consumption. $P_{S}$ is the power consumption at sleep mode. $N_{T R X}$ is the number of transceiver chains. $t_{T x}, t_{R x}$, $t_{0}$ and $t_{s}$ are time of eNBs in transmit, receive, idle and sleep modes. The parameters based on the 2010 state-of-art estimation are summarized in Table I.

Table I. Energy Parameters

\begin{tabular}{|l|l|}
\hline Parameters & Values \\
\hline Number of Transceiver Chains $N_{T R X}$ & 2 \\
\hline Load dependent slope $\Delta_{P}$ & 2.6 \\
\hline Maximum output power $P_{\text {out }}$ & $5 \mathrm{~W}$ \\
\hline Power consumption at sleep mode $P_{S}$ & $39 \mathrm{~W}$ \\
\hline Power consumption without RF $P_{0}$ & $56 \mathrm{~W}$ \\
\hline
\end{tabular}

Using a data link level simulation in MATLAB, this paper examines the system level performance which could be obtained from the developed topology management algorithms. Data traffic is modelled on both downlinks and uplinks, with other traffic types likely to have similar relative performance. This is modelled using a file transfer traffic model, which simulates a succession of packets delivered in the network [15]. 
The inter-arrival time and file size are modelled as long-tailed Pareto distribution.

The transmission time of a file is affected by the link data rate, which is determined by the bandwidth efficiency at a given SINR level. The SINR at a receiver is given by

$$
\gamma=\frac{P_{T x} G_{T x} G_{R x}\left(P L_{T x R x}\right)^{-1}}{n+\sum_{T x^{\prime}=0, T x^{\prime} \neq \neq x}^{N} P_{T x^{\prime}} G_{T x^{\prime}} G_{R x}\left(P L_{T x^{\prime} R x}\right)^{-1}}
$$

where $T x$ and $R x$ are the transceiver pair and $T x^{\prime}$ denotes other transmitters in the system that could potentially cause interference. $P$ is the transmit power, which is allocated equality on each channel. $n$ is the thermal noise. $G$ is the antenna gain. $P L$ is the path loss. The free space propagation model with $8 \mathrm{~dB}$ log-normal shadowing is used on aerial links, based on the aerial platform study in [16]. The WINNER II C1 urban microcell model is used on terrestrial links [17].

The truncated Shannon model [18] is used to determine the link data rate at a particular SINR level, which is a representative of bandwidth efficiency that can be achieved in practice given an adaptive modulation and coding codeset:

$$
\rho=\left\{\begin{array}{cc}
0 & \gamma<\gamma_{\mathrm{MIN}} \\
\alpha W \log _{2}(1+\gamma) & , \gamma_{\mathrm{MIN}} \leq \gamma \leq \gamma_{\mathrm{MAX}} \\
\alpha W \log _{2}\left(1+\gamma_{\mathrm{MAX}}\right) & \gamma>\gamma_{\mathrm{MAX}}
\end{array}\right.
$$

where $W$ is the channel bandwidth, $\alpha$ is the implementation loss of Shannon bound, $\gamma_{\text {MIN }}$ is the minimum SINR requirement of a communication link and $\gamma_{\text {MAX }}$ denotes the SINR that contributes to the maximum data rate.

\section{TRANSFER LEARNING BASED TOPOLOGY MANAGEMENT}

In this section, we study topology management in two aspects: user association and BS switching operation, with QoS aware algorithms developed. Transfer learning is introduced to improve performance end reduce complexity, by transferring statistical experience from spectrum assignment.

\section{A. User Association Algorithms}

BS user association has been specified in LTE as cell selection and reselection, to allow UEs to search for a suitable cell to camp on in idle or connected modes [19]. This is achieved through an evaluation of Reference Signal Received Power (RSRP) between eNBs and UEs. However, the best signal eNB may suffer from traffic congestion or interference. In LTE, this is controlled by inter-cell interference coordination (ICIC), which allows an overloaded eNBs to handover UEs to adjacent or overlaid cells. However, the traffic offloading capability of a cell is highly dynamic affected by interference in a spectrum sharing scenario. Moreover, ICIC may incur excessive information exchange in $5 \mathrm{G}$ dense small cell networks. In this paper, we extend the functionality of user association to a network level, which allows it to considers multiple impacts including signal, interference, bottleneck, traffic congestion, spectrum availability, etc.

\section{1) QoS aware User Association}

In order to make the user association process fully distributed, we propose a QoS aware user association approach. The LTE network allows a single cell to utilize the entire spectrum band, and the future $5 \mathrm{G}$ network is likely to enable full spectrum sharing between cells. In this context, inter-cell interference can cause a data file to be blocked initially or interrupted during transmission when SINR drops below the minimum level for communications. Such blocked and interrupted files will be scheduled for retransmission after a random back off time, which has dominating impact on system performance. Therefore, the probability of retransmissions effectively characterises the radio environment (interference, traffic congestion) and QoS performance (delay, throughput), which can be monitored by the eNB on a temporal basis.

In the user association procedure, RSRP is used to inform the UE of a list of eNBs that can provide service. The active eNBs are ranked in a list $b^{s}$ by the UE based on RSRP (denoted as $\gamma$ ). For $\forall i \in b^{s}$, when $\exists b^{s}(i+1)$ :

$$
\gamma\left(b^{s}(i)\right) \geq \gamma\left(b^{s}(i+1)\right) \& \forall \gamma\left(b^{s}(i)\right) \geq \gamma_{\mathrm{MIN}}
$$

The active eNB list $b^{s}$ contains AeNBs $b_{a}^{s}$ and TeNBs $b_{t}^{s}$, which use equally divided spectrum where no interference exists between the two system. However, the capacity provided from AeNBs can be highly limited because they have lower spectrum reuse capability. The aerial links provide better signal power than the terrestrial links in most of the areas where UEs are far from TeNBs. In this context, the RSRP based user association algorithm can cause the AeNBs to be overloaded.

In order to effectively control the traffic load on AeNBs and also the interference between TeNBs, the QoS aware user association algorithm allows the UEs to compare the retransmission probability $P_{r}$ on the AeNB $b_{a}^{s}$ with that on all other TeNBs $b_{t}^{s}$ following their order in $b^{s}$. In a list of TeNBs that have lower $P_{r}$ than the AeNB, the one with highest RSRP can be selected. The same criterion applies to the AeNBs. For $\forall i \in b^{s}$, the selected TeNB $b_{t}^{s}(s)$ or AeNB $b_{a}^{s}(s)$ follows

$$
\begin{aligned}
& b_{t}^{s}(s): P_{r}\left(b_{t}^{s}(s)\right) \leq P_{r}\left(b_{a}^{s}(i)\right) \& \gamma\left(b_{t}^{s}(s)\right) \geq \gamma\left(b_{t}^{s}(i)\right) \\
& b_{a}^{s}(s): P_{r}\left(b_{a}^{s}(s)\right) \leq P_{r}\left(b_{t}^{s}(i)\right) \& \gamma\left(b_{a}^{s}(s)\right) \geq \gamma\left(b_{a}^{s}(i)\right)
\end{aligned}
$$

In most cases, there are more TeNBs than AeNBs in the candidate list $b^{s}$, which thus has higher probability to be selected. The traffic load in each cell is expected to match with its reuse capability.

\section{2) Transfer Learning based User Association}

Traffic and interference profile in a RAN may have a large amount of short term variation, which causes the interim QoS parameter to be highly unstable. In conventional QoS monitoring approaches, the system cannot evaluate the stability of the radio environment, and intelligently uses the instantaneous and historical information to stabilize or change the decisions. To overcome this issue, we introduce cognitive engines at the eNBs to enable intelligent user association with dynamic spectrum assignment using reinforcement learning.

As described in the system model, two spectrum bands are shared by all AeNBs and TeNBs, respectively. We hereby apply a single state Q learning algorithm developed in our previous work [20] for cognitive spectrum assignment. Specifically, the eNBs are implemented with a knowledgebase that stores a set of channel-associated $Q$ values. A learning iteration is triggered by a file arrival on either the uplink or 
downlink. The eNB makes decision on channel selection according to a defined decision making policy:

$$
a_{k}=\pi(a) \in \arg \max Q_{a}(t)
$$

where the channel $a_{k}$ with maximum $\mathrm{Q}$ value in the spectrum pool $a$ is selected at iteration $t$. A successfully selected channel is then assigned to the file for data transmission. In the event of a failure selection, this process will be carried out iteratively until no channel is available. A learning function is then used to update the $\mathrm{Q}$ array in the event a file is either delivered or rescheduled for transmission:

$$
Q(t)=(1-\lambda) Q(t-1)+\lambda R, \lambda \in(0,1)
$$

where $\lambda$ is the learning rate controlling the convergence speed. $R$ is the reward that reinforces the $\mathrm{Q}$ array based on the outcome of decision making:

$R_{k}=\left\{\begin{array}{c}1 \text { (positive reinforcement): Success Iteration } \\ -1 \text { (negative reinforcement): Failure Iteration }\end{array}\right.$

The algorithm has been validated in [20] where effective spectrum partitioning and QoS are delivered.

Spectrum assignment and user association are two highly related domains with extensive similarities. They can be triggered in the same iteration when a data file arrives, affected by success or failure decisions, and determine the QoS. We thus develop a transfer learning function that transfers the expert knowledge learnt from the source domain (spectrum assignment) to the target domain (user association). The $\mathrm{Q}$ values in spectrum assignment contain the information of success or failure file transmission on each channel. By aggregating a $\mathrm{Q}$ array appropriately, we can have a new $\mathrm{Q}$ value that provides the QoS information of the whole cell.

In the spectrum assignment domain, the $Q$ values in an array contain different amount of learnt information, depending on the number of iterations that their associated channels have been selected. However, this cannot be identified in the single state Q learning where the Q values fluctuate between the reward values. Therefore in transfer learning, we normalize the $Q$ values and aggregate them proportionally based on their number of iterations, which is expected to differentiate the learnt information. By defining the number of iterations as $N$, a $\mathrm{Q}$ value in the user association domain, associated with eNB $b(i)$, can be calculated from:

$$
\forall k \in a: Q_{b(i)}^{U A}=\sum Q_{b(i)}^{S A}(k) \frac{N_{b(i)}^{S A}(k)}{\sum N_{b(i)}^{S A}(k)}
$$

On the arrival of a file, the UE collects $Q^{U A}$ from multiple eNBs with RSRP satisfying the SINR threshold, and makes decisions on eNB selection based on the newly formulated $\mathrm{Q}$ array. Q learning is then carried out for spectrum assignment. This algorithm uses one cognitive engine to solve two different learning tasks, which effectively reduce system complexity.

In order to use this algorithm for load management purposes in the two layer system, we apply the same user association procedure in the QoS aware approach, by applying the Q array to the selection criteria. For $\forall i \in b^{s}$, the selected $b_{t}^{s}(s)$ or $b_{a}^{s}(s)$ is as follows
$b_{t}^{S}(s): Q^{U A}\left(b_{t}^{s}(s)\right) \leq Q^{U A}\left(b_{a}^{s}(i)\right) \& \gamma\left(b_{t}^{s}(s)\right) \geq \gamma\left(b_{t}^{s}(i)\right)$

$b_{a}^{s}(s): Q^{U A}\left(b_{a}^{s}(s)\right) \leq Q^{U A}\left(b_{t}^{s}(i)\right) \& \gamma\left(b_{a}^{s}(s)\right) \geq \gamma\left(b_{a}^{s}(i)\right)$

The integrated user association and spectrum assignment process with transfer learning algorithm is shown below. By exploiting the performance in RRM layer, this algorithm has the potential to achieve effective load balancing, congestion control, interference management, and ultimately improves QoS without complex optimization algorithm.

Algorithm 1. Transfer Learning based User Association and Spectrum Assignment

When a new file arrives on uplink or downlink

1. UE evaluates RSRP of adjacent eNBs, achieves a candidate eNB list $b^{s}$ based on (4)

2. UE sends connection request to eNBs in $b^{s}$

3. eNB obtains $Q_{b(i)}^{S A}$ from knowledgebase, calculate $Q_{b(i)}^{U A}$ based on transfer learning function (9) and send to UE

4. UE generates a $\mathrm{Q}$ array based on $\mathrm{Q}$ values sent from all eNBs in $b^{s}$, makes decisions of eNB selection based on (10)

initialize $s \leftarrow \emptyset, i \leftarrow 1$

while $s=\emptyset$, do

if $Q\left(b_{t}^{s}(i)\right) \leq Q\left(b_{a}^{s}(j)\right), \forall j \in b_{a}^{s}$

then $s \leftarrow b_{t}^{s}(i)$;

else if $\nexists b_{t}^{s}(i+1)$

then $s \leftarrow b_{a}^{s}(1)$;

$$
\text { end if }
$$

\section{end while}

5. UE associated with eNB $s$

6. eNB $s$ makes decisions on channel selection based on (6), assigns to UE for file transmission

7. eNB $s$ observes the outcome based on (7)

8. eNB $s$ updates $\mathrm{Q}$ array $Q_{b(s)}^{S A}$ based on (8)

\section{B. QoS aware BS Switching Operation}

Network virtualization has been proposed as a candidate technique in $5 \mathrm{G}$ to simplify the functional architecture. In this context, a virtualized network controller is introduced to monitor the QoS in a localized area and manage the network topology. The purpose of QoS aware BS switching approach is to maximize the sleep mode operation while still providing effective QoS within a tolerance range from system requirement configuration. To achieve this, the network controller regularly collects the retransmission probability from all the activated TeNBs $P_{r}\left(b_{t}\right)$, and calculates the system level $P_{r}$. A retransmission tolerance $\left[P_{r}^{l}, P_{r}^{u}\right]$ is applied to switch TeNBs between active and sleep modes.

In order to maximize the user traffic that active TeNBs can serve, the network controller monitors average loading level $L($.$) of all active TeNBs, and makes decisions of switching on$ $b_{t}^{O N}$ or off $b_{t}^{O F F}$ a TeNB according to the following criteria:

$$
\forall i \in b_{t}: \begin{aligned}
& L\left(b_{t}^{O N}\right) \geq L\left(b_{t}(i)\right) \& P_{r}>P_{r}^{u} \\
& L\left(b_{t}^{O F F}\right) \leq L\left(b_{t}(i)\right) \& P_{r}<P_{r}^{l}
\end{aligned}
$$




\section{System Simulation AND PERFormance EVAlUation}

In this section, a system level simulation of the example aerial-terrestrial broadband access network architecture is demonstrated to validate transfer learning and QoS aware user association and BS operation algorithms. The simulator is based on the system model described in Section II and key parameters listed in TABLE II. It has been studied in [21] that the learning rates higher than 0.05 has few system impacts.

TABLE II. SiMULATION PARAMETERS

\begin{tabular}{|c|c|c|}
\hline \multicolumn{2}{|c|}{ Parameters } & Values \\
\hline \multicolumn{2}{|c|}{ Carrier Frequency } & $2.6 \mathrm{GHz}$ \\
\hline \multicolumn{2}{|c|}{ Bandwidth } & AeNB: $10 \mathrm{MHz} ;$ TeNB: $10 \mathrm{MHz}$ \\
\hline \multicolumn{2}{|c|}{ Number of Channels } & AeNB: $20 ;$ TeNB: 20 \\
\hline \multicolumn{2}{|c|}{ Transmit Power $P_{T x}$} & AeNB/TeNB: $37 \mathrm{dBm}$; UE: $23 \mathrm{dBm}$ \\
\hline \multicolumn{2}{|c|}{ Thermal Noise } & $-174 \mathrm{dBm} / \mathrm{Hz}$ \\
\hline \multirow{2}{*}{$\begin{array}{l}\text { Traffic } \\
\text { Model }\end{array}$} & Inter-arrival time & Pareto distribution: $\alpha=4$ \\
\hline & File size & $100 \mathrm{kB}$ \\
\hline \multicolumn{2}{|c|}{ Antenna profile } & Omni-directional \\
\hline \multicolumn{2}{|l|}{ Height } & AeNB: $100 \mathrm{~m}$; TeNB: $25 \mathrm{~m}$; UE: $1.5 \mathrm{~m}$ \\
\hline \multicolumn{2}{|c|}{ QoS tolerance } & Retransmission Probability: [5\%,10\%] \\
\hline \multicolumn{2}{|c|}{ Learning rate } & 0.1 \\
\hline \multicolumn{2}{|c|}{ Effective SINR $\left[\gamma_{\text {MIN }}, \gamma_{\text {MAX }}\right]$} & {$[1.8,21]$} \\
\hline
\end{tabular}

The percentage of eNBs in sleep mode when applying BS switching operation (SO) is demonstrated in Fig. 3. A comparison between the transfer learning (TL), QoS and RSRP based user association algorithms (UA) shows that the load management performance has large impact on network scale and energy efficiency under the same switching criteria.

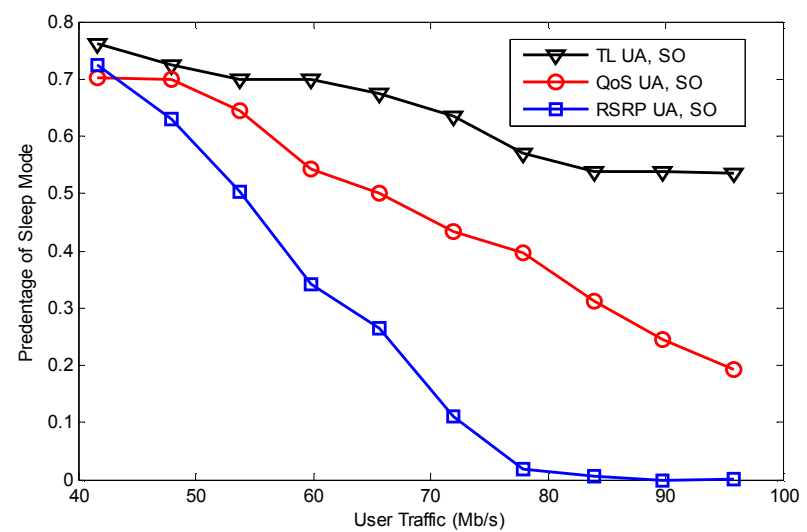

Fig. 3. Percentage of Base Stations in Sleep Mode

It can be observed that in low traffic levels, around $80 \%$ of the eNBs are switched off, namely the 2 AeNBs can effectively support the network traffic. The conventional RSRP algorithm requires significantly more TeNBs to serve the network as user traffic increases, reaching full deployment at $85 \mathrm{Mb} / \mathrm{s}$. On the other hand, the QoS algorithm largely reduces the scale of the network by up to $40 \%$, which is achieved through effective load management between AeNBs and TeNBs. The transfer learning algorithm is demonstrated to further reduce the number of active eNBs, by up to $30 \%$ more than the QoS algorithm and $60 \%$ more than the RSRP algorithm. It can be concluded from here that the historical learnt knowledge from spectrum assignment can effectively improve decision making on user association compared to interim QoS information.
The average power consumption per eNB is demonstrated in Fig. 4, which is evaluated through the energy model presented in Section II. It is demonstrated that the QoS aware BS switching operation algorithm significantly reduce the power consumption of by up to $30 \mathrm{~W}$ at low traffic levels. In user association, the QoS algorithm achieves $15 \mathrm{~W}$ more energy saving than the RSRP algorithm at $80 \mathrm{Mb} / \mathrm{s}$ traffic, and the transfer learning algorithm further saves $15 \mathrm{~W}$ at $95 \mathrm{Mb} / \mathrm{s}$. Compared with Fig. 3 we can conclude that significant energy savings can be achieved by using effective sleep mode operation on BSs. Furthermore, it can be seen from the same figure that without sleep mode operation, the transfer learning and QoS based user association algorithm has slightly higher power consumption than the and RSRP algorithm. This is because some UEs are associated with long distance eNBs, which increases the average transmission power consumption. However, this can be effectively compensated from sleep mode energy saving as shown in the figure.

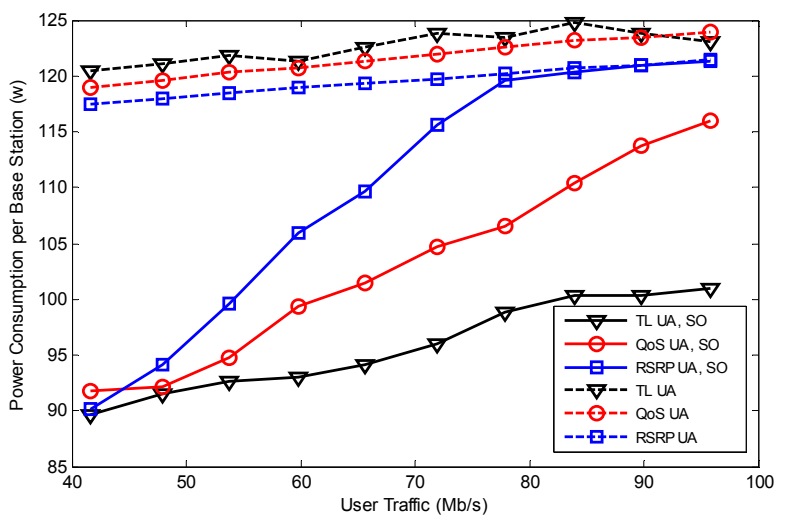

Fig. 4. Average Power Consumption per Base Station

Fig. 5 presents the probability of retransmissions, which is used as a reference QoS parameter for user association and switching operation. Firstly, by comparing the performance of three user association algorithms in the full network scenario, we can see that the QoS and transfer learning algorithms largely reduce the retransmission probability compared to the RSRP algorithm. Such QoS improvement effectively helps to reduce energy in BS switching operation. Moreover, transfer learning further reduces retransmission by $5 \%$ at high traffic levels, but with significantly greater energy savings achieved compared with the gain from QoS algorithm.

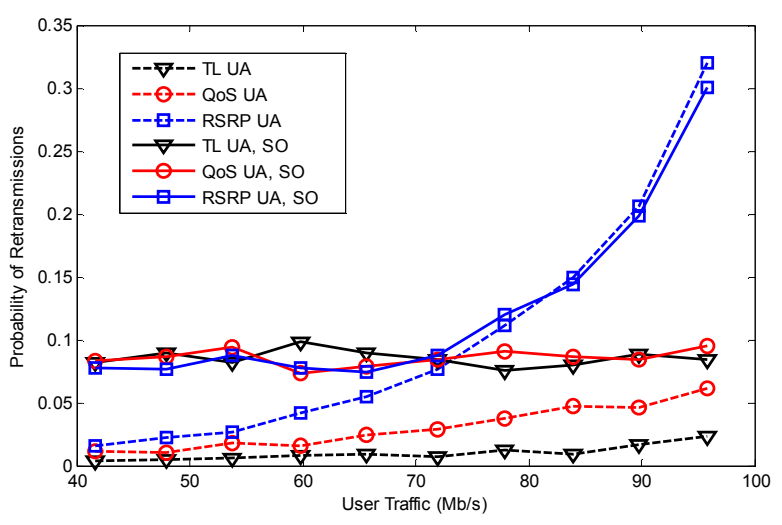

Fig. 5. Probability of Retransmissions 
In the same figure we can see that, the BS switching operation algorithm achieves a system retransmission probability in the range of $[5 \%, 10 \%]$ on all user association algorithms before all eNBs are activated, which satisfies the predefined QoS configuration from system requirement in TABLE II. It can be concluded that the QoS aware switching algorithm effectively controls the QoS degradation affected by reduced number of BSs, with expected QoS provided.

The system delay performance is demonstrated in Fig. 6. Similarly the delay level of BS switching operation algorithms falls within [0.1s, $0.2 \mathrm{~s}$ ] over a wide range of traffic levels by switching off TeNBs. At low traffic levels the delay is slightly higher because of the low data rate on the aerial link. The QoS aware and transfer learning based use association algorithms largely improve the delay performance at high traffic levels by more effective load management. Further delay reduction is also achieved by transfer learning at high traffic levels while providing significant energy saving.

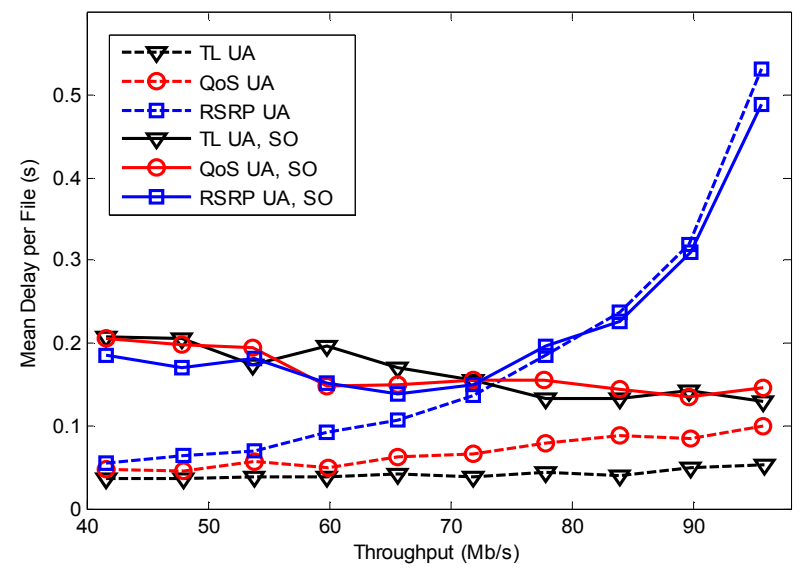

Fig. 6. System Delay

\section{CONCLUSION}

This paper studied the use of transfer learning to deal with base station user association, using a QoS aware base station switching operation approach to reduce energy consumption and improve QoS in an aerial-terrestrial broadband access network. A cognitive engine has been designed to provide intelligent self-organized spectrum assignment and user association at eNB, using a distributed Q learning algorithm. A transfer learning algorithm has been developed which effectively utilizes historically learnt knowledge from spectrum assignment to make decisions on user association. Furthermore, a tolerance range of system QoS has been used to dynamically switch eNBs between active and sleep modes to save energy, under the support of load management from user association.

System level simulations from practical configurations demonstrate that the transfer learning algorithm places $30 \%$ to $60 \%$ more TeNBs into sleep mode with significant power savings compared to QoS and signal based algorithms, respectively. Significant QoS improvement is achieved by effective load management from the QoS aware and transfer learning based user association. Furthermore, the base station switching operation algorithm reduces the energy consumption considerably while providing adequate QoS.

\section{ACKNOWLEDGEMENT}

This work has been funded by the ABSOLUTE Project (FP7-ICT-2011-8-318632), which receives funding from $7^{\text {th }}$ Framework Programme of European Commission.

\section{REFERENCES}

[1] S. Dehghan and R. Steele, "Small cell city," Communications Magazine, IEEE, vol. 35, pp. 52-59, 1997.

[2] Net!Works European Technology Platform: Expert Working Group on Spectrum Crunch - White Paper, June 2012. 5g-ppp.eu/white-papers

[3] Z. Niu, "TANGO: traffic-aware network planning and green operation," Wireless Communications, IEEE, vol. 18, pp. 25-29.

[4] J. Hoydis, M. Kobayashi, and M. Debbah, "Green Small-Cell Networks," Vehicular Technology Magazine, IEEE, vol. 6, pp. 37-43.

[5] FP7 EARTH D2.3: Energy efficiency analysis of the reference systems, areas of improvements and target breakdown. www.ict-earth.eu

[6] F. Richter, A. J. Fehske, and G. P. Fettweis, "Energy Efficiency Aspects of Base Station Deployment Strategies for Cellular Networks," in Vehicular Technology Conference Fall (VTC 2009-Fall), 2009 IEEE 70th, 2009, pp. 1-5.

[7] P. Gonzalez-Brevis, J. Gondzio, F. Yijia, H. V. Poor, J. Thompson, I. Krikidis, et al., "Base Station Location Optimization for Minimal Energy Consumption in Wireless Networks," in Vehicular Technology Conference (VTC Spring), 2011 IEEE 73rd, 2011, pp. 1-5.

[8] Y. Wu and Z. Niu, "Energy Efficient Base Station Deployment in Green Cellular Networks with Traffic Variations," in Communications in China (ICCC), 2012 1st IEEE International Conference on, pp. 399-404.

[9] Y. Han, D. Grace, and P. Mitchell, "Energy efficient topology management for beyond next generation mobile broadband systems," in Wireless Communication Systems (ISWCS), 2012 International Symposium on, pp. 331-335.

[10] S. Kyuho, K. Hongseok, Y. Yung, and B. Krishnamachari, "Base Station Operation and User Association Mechanisms for Energy-Delay Tradeoffs in Green Cellular Networks," Selected Areas in Communications, IEEE Journal on, vol. 29, pp. 1525-1536, 2011.

[11] B. Rengarajan, G. Rizzo, M. Ajmone Marsan, and B. Furletti, "QoSaware greening of interference-limited cellular networks," in World of Wireless, Mobile and Multimedia Networks (WoWMoM), 2013 IEEE 14th International Symposium and Workshops on a, 2013, pp. 1-9.

[12] P. Sinno Jialin and Y. Qiang, "A Survey on Transfer Learning," Knowledge and Data Engineering, IEEE Transactions on, vol. 22, pp. 1345-1359.

[13] R. Li, Z. Zhao, X. Chen, P. J., and H. Zhang, "TACT: A Transfer ActorCritic Learning Framework for Energy Saving in Cellular Radio Access Networks," Wireless Communications, IEEE Transactions on, vol. 13, pp. 2000-2011, 2014

[14] FP7-ABSOLUTE. Aerial Base Stations with Opportunistic Links for Unexpected \& Temporary Events. http://www.absolute-project.eu

[15] J. D. Chimeh, M. Hakkak, and S. A. Alavian, "Internet Traffic and Capacity Evaluation in UMTS Downlink," in Future Generation Communication and Networking, 2007, pp. 547-552.

[16] FP7-ICT-ABSOLUTE/D2.3.1: Aerial Platforms Study. Available: www.absolute-project.eu

[17] P. Kyösti, J. Meinila, L. Hentila, X. Zhao, and T. Jamsa, WINNER II Channel Models v1.2, IST-WINNER D1.1.2, 2007.

[18] A. Papadogiannis and A. G. Burr, "Multi-beam assisted MIMO - A novel approach to fixed beamforming," in Future Network \& Mobile Summit (FutureNetw), 2011, pp. 1-8.

[19] 3GPP TS 25.304: User Equpment (UE) procedures in idle mode and procedures for cell reselection in connected mode, v. 12.1.0. Available: www.3gpp.org/Releases

[20] Q. Zhao, T. Jiang, N. Morozs, D. Grace, and T. Clarke, "Transfer Learning: A Paradigm for Dynamic Spectrum and Topology Management in Flexible Architectures," in IEEE 78th Vehicular Technology Conference September 2013.

[21] N. Morozs, T. Clarke, and D. Grace, "A Novel Adaptive Call Admission Control Scheme for Distributed Reinforcement Learning Based Dynamic Spectrum Access in Cellular Networks," in Wireless Communication Systems (ISWCS 2013), Proceedings of the Tenth International Symposium on, 2013, pp. 1-5. 